$\exists \int$ Journal of Logic \& Analysis 4:1 (2012) 1-14

ISSN 1759-9008

\title{
Convergence results for function spaces over o-minimal structures
}

\author{
MARGARET E M THOMAS
}

\begin{abstract}
We begin the development of a theory of Banach spaces in the definable setting of o-minimal structures. We outline several results which develop the theory of compact embeddings for explicitly given function spaces. One aim is to explain the substantive underpinnings of an important observation used in the proof of the Reparameterization Theorem of Pila and Wilkie in [2]. We place this observation in the broader context of our theory and demonstrate how it may be refined further.
\end{abstract}

2000 Mathematics Subject Classification 03C64 (primary); 46B99 (secondary)

Keywords: o-minimal structures, parameterization, Banach spaces

\section{Introduction}

The goal of this note is to introduce some Banach space theory in the definable context of o-minimal expansions of real closed fields. The development of this theory was originally motivated by the search for the proof of the Reparameterization Theorem of Pila and Wilkie (Theorems 2.3 and 2.5 of [2]). An important observation made during the course of their proof is that reparameterizations converge to reparameterizations having a sufficient degree of differentiability. This property is a consequence of the theory developed in this paper, in particular Corollary 3.7, a fact explained by Remark 4.1 of [2]. It is our aim in this note to provide an introductory exposition of this theory, outlining results which go beyond Remark 4.1. This will not only furnish the substance of Remark 4.1 (and hence flesh out the details of this essential step in the proof of the Reparameterization Theorem), but also provide some details of a theory which we believe to be interesting in its own right, laying the groundwork for what we hope will be a fruitful area of future analysis.

We have not yet developed a completely general form of this Banach space theory, but rather focus here on certain explicitly given spaces of definable functions. We present definable notions of convergence and completeness for such spaces and outline a theory of compact embeddings in this context. All definitions are outlined in Section 2, with our results following in Section 3. We conclude in Section 4 with some remarks concerning possible directions in which this theory could be developed further. 


\section{Definable Banach Spaces}

\subsection{Definitions}

Let us first fix some notation and present some relevant theory from functional analysis, amended appropriately for our setting. Throughout, $\mathcal{M}=\langle M ;+, \cdot,-; 0,1 ;<; \ldots\rangle$ will denote an o-minimal expansion of a real closed field and $X$ will denote a vector space over $\mathcal{M}$. For an exposition of the fundamental properties of o-minimal structures, some of which will be used extensively in Section 3, please see, for example, van den Dries [1].

We define an $\mathcal{M}$-norm on $X$ to be a function $\|\cdot\|_{X}: X \rightarrow M$ which satisfies the usual norm axioms, i.e. positivity, positive definiteness, the triangle inequality and positive homogeneity. The presence of an $\mathcal{M}$-norm acting on a space $X$ induces a $\mathcal{M}$-norm topology on that space. As expected, this is given by basic open sets of the form $\mathbf{B}_{X}^{\epsilon}(x):=\left\{y \in X \mid\|x-y\|_{X}<\epsilon\right\}$. We denote the closed unit ball of an $\mathcal{M}$-normed space $\left(X,\|\cdot\|_{X}\right)$ by $\mathbf{B}_{X}:=\left\{x \in X \mid\|x\|_{X} \leq 1\right\}$.

Now that we have established these definitions, since $\mathcal{M}$ will be fixed throughout this paper, we shall also simplify our terminology throughout to refer to norms, rather than $\mathcal{M}$-norms, without any loss of clarity.

\subsection{Function Spaces}

We shall be especially concerned here with those spaces $X$ which are function spaces over $\mathcal{M}$. These are topological vector spaces whose elements are themselves functions defined on a fixed, bounded domain $Y$ (i.e. on some open, definable $Y \subseteq \prod_{i=1}^{n}\left[-S_{i}, S_{i}\right]$, for some $\left.S_{1}, \ldots, S_{n} \in M\right)$. We present a few important examples of such spaces, together with their norms.

Examples 2.1 Suppose that $Y$ is a bounded domain in $M^{n}$, for some $n \geq 1$.

$\mathcal{B}(Y)$, the space of all definable, bounded maps $f: Y \rightarrow M$, is a normed space when equipped with the supremum norm, defined thus:

$$
\|f\|_{\infty}:=\sup _{y \in Y}\{|f(y)|\} .
$$

$\mathcal{C}(Y)$, the space of all definable, continuous, bounded, $M$-valued functions on $\mathrm{Y}$, is a subspace of $\mathcal{B}(Y)$, and hence is a normed space when equipped with the supremum norm. 
$\mathcal{C}^{r}(Y)$, the space of all definable, $r$-times continuously differentiable, bounded, $M-$ valued functions on $Y$, which have their first $r$ derivatives bounded, is a normed space when equipped with the norm

$$
\|f\|_{r}:=\max _{0 \leq t \leq r}\left\{\left\|f^{(t)}\right\|_{\infty}\right\}
$$

Define a map $\operatorname{Lip}(\cdot)$, from $\mathcal{C}^{\operatorname{Lip}}(Y)$, the space of all definable, bounded, Lipschitz continuous, $M$-valued functions on $Y$, into $M$, by

$$
\operatorname{Lip}(f):=\sup _{\substack{x \neq y \\ x, y \in Y}}\left\{\frac{|f(x)-f(y)|}{|x-y|}\right\} .
$$

Then $\operatorname{Lip}(f)$ is, for a Lipschitz continuous function $f$, the least constant which can witness the Lipschitz continuity of $f$. Let us define $\|f\|_{\text {Lip }}:=\operatorname{Lip}(f)$. This is not a norm on the space $\mathcal{C}^{\operatorname{Lip}}(Y)$, as it does not satisfy the axiom of positive definiteness. However, $\mathcal{C}^{r \text { Lip }}(Y)$, the space of all definable, $r$-times continuously differentiable, bounded, $M-$ valued functions on $Y$, which have bounded first $r$ derivatives and Lipschitz continuous $r$ th derivative, is a normed space when equipped with the norm

$$
\|f\|_{r, \text { Lip }}:=\max \left\{\|f\|_{r},\left\|f^{(r)}\right\|_{\text {Lip }}\right\} \text {. }
$$

\subsection{Definable Convergence}

Here we outline definable notions of convergence and completeness within these function spaces. We express these notions in terms of definable curves.

Definitions 2.2 Let $Y$ be a bounded domain in $M^{n}$, for some $n \geq 1$, and let $X$ be a subspace of $\mathcal{B}(Y)$ equipped with the norm $\|\cdot\|_{X}$. A definable curve in $X$ is a family $\mathcal{F}:=\left\{F_{u}: Y \rightarrow M \mid u \in(0,1)\right\}$ of functions in $\mathcal{B}(Y)$ such that

(i) $F_{u} \in X$, for all $u \in(0,1)$;

(ii) the map from $(0,1) \times Y$ to $M$ given by $\langle u, y\rangle \mapsto F_{u}(y)$ is definable;

(iii) the function $u \mapsto F_{u}$ is continuous with respect to the usual $(|\cdot|-)$ topology on $(0,1)$ and the $\|\cdot\|_{X}$-topology on $X$.

Such a curve converges as $u \rightarrow 0^{+}$if and only if there exists $F_{0} \in X$ such that $\left\|F_{u}-F_{0}\right\|_{X} \rightarrow 0$ as $u \rightarrow 0^{+}$. 
Remark One generalization of the usual definition of sequential convergence is that of nets, which are limits over directed sets. Applying the definition of o-minimality, definable curves could be viewed in this context as "definable nets" for which the directed set is $(0,1)$. This gives us that the above is an appropriate definition of convergence in spaces of definable functions from the point of view of functional analysis as well as that of algebraic geometry.

What should be clear is that many familiar definitions of convergence will naturally translate to definable statements expressed in terms of convergence along definable curves. However, as definable curves are definable maps, and so the notion of convergence along them is a definable one, generalizations that can be made about convergence in an $\mathcal{M}$-normed space will, by necessity, be restricted to definable notions. For example, let us make the following two definitions.

Definition 2.3 Let $Y$ be a bounded domain in $M^{n}$, for some $n \geq 1$, and let $X$ be a vector subspace of $\mathcal{B}(Y)$ equipped with the norm $\|\cdot\|_{X}$. A definable curve $\mathcal{F}:=\left\{F_{u}: Y \rightarrow M \mid u \in(0,1)\right\}$ is said to be Cauchy as $u \rightarrow 0^{+}$if and only if $\left\|F_{u_{1}}-F_{u_{2}}\right\|_{X} \rightarrow 0$ as $u_{1}, u_{2} \rightarrow 0^{+}$.

Remark Note that any curve $\mathcal{F}$ which is Cauchy is also bounded: by the continuity of the function $u \mapsto F_{u}$ on $(0,1)$, we need only see that this function does not become unbounded as $u \rightarrow 0^{+}$. For this we may use a simple inequality argument, as follows. Since $\mathcal{F}$ is Cauchy, we have that, for all $\epsilon>0$, there exists $\delta>0$ such that, for all $u \in(0, \delta]$, we have $\left\|F_{u}-F_{\delta}\right\|_{X}<\epsilon$. Hence, for all $u \in(0, \delta]$, it also follows that $\left\|F_{u}\right\|_{X} \leq\left\|F_{\delta}\right\|_{X}+\epsilon$. Thus $\mathcal{F}$ is bounded as $u \rightarrow 0^{+}$.

Definition 2.4 An $\mathcal{M}$-normed function space $X$ is said to be $\mathcal{M}$-definably complete if every definable curve in $X$ which is Cauchy also converges.

So, for our definable notion of completeness, we do not have that every definable curve in $X$ converges, but specifically require that definable Cauchy curves in $X$ do.

When $X$ is a complete, normed space, in the conventional sense, $X$ is said to be a Banach space. However, given our restriction only to convergence along definable curves in spaces of bounded, definable functions on bounded domains, we shall call these $\mathcal{M}$-definably complete, $\mathcal{M}$-normed spaces of definable functions $\mathcal{M}$-Banach spaces.

Examples 2.5 The following are all examples of $\mathcal{M}$-Banach spaces. 
The domain $M$ of our o-minimal expansion $\mathcal{M}$ of a real closed field is itself an $\mathcal{M}$-Banach space when equipped with the usual norm $|\cdot|$, if all elements of $M$ are considered as constant functions on $Y$, for some bounded domain $Y$ in $M^{n}$, with $n \geq 1$.

The space of bounded, definable functions $\mathcal{B}(Y)$ is complete for $\|\cdot\|_{\infty}$ and thus is an $\mathcal{M}$-Banach space, by the following argument.

Let $\mathcal{F}:=\left\{F_{u}: Y \rightarrow M \mid u \in(0,1)\right\}$ be a definable Cauchy curve in $\mathcal{B}(Y)$. On $Y$ we may define the pointwise limit function $F_{0}(y):=\lim _{u \rightarrow 0^{+}} F_{u}(y)$, since this limit exists, for all $y \in Y$, by o-minimality; the function $F_{0}$ takes values in $M \cup\{ \pm \infty\}$.

Since $\mathcal{F}$ is Cauchy, $\left\|F_{u}\right\|_{\infty}$ is bounded, by $C$, say. So

$$
\left\|F_{0}\right\|_{\infty}=\sup _{y \in Y}\left|\lim _{u \rightarrow 0^{+}} F_{u}(y)\right| \leq \sup _{u \in(0,1)}\left\|F_{u}\right\|_{\infty} \leq C .
$$

Thus $F_{0}$ is bounded, and hence $F_{0} \in \mathcal{B}(Y)$.

Finally, let $\epsilon>0$ and observe that, since $\mathcal{F}$ is Cauchy, there exists $\delta>0$ such that, if $u_{1}, u_{2} \in(0, \delta)$, then $\left\|F_{u_{1}}-F_{u_{2}}\right\|_{\infty}<\epsilon$. Therefore, for all $y \in Y$, if $u_{1}, u_{2} \in(0, \delta)$, then $\left|F_{u_{1}}(y)-F_{u_{2}}(y)\right|<\epsilon$. Hence, if $u_{1} \in(0, \delta),\left|F_{u_{1}}(y)-F_{0}(y)\right|=$ $\lim _{u_{2} \rightarrow 0^{+}}\left|F_{u_{1}}(y)-F_{u_{2}}(y)\right| \leq \epsilon$. Since this bound is independent of $y$, we have that, if $u_{1} \in(0, \delta)$, then $\left\|F_{u_{1}}-F_{0}\right\|_{\infty} \leq \epsilon$, and hence $\mathcal{F}$ converges to $F_{0}$ in the supremum norm as $u \rightarrow 0^{+}$.

Any closed, linear subspace of an $\mathcal{M}$-Banach space is $\mathcal{M}$-definably complete for the same norm and thus is itself an $\mathcal{M}$-Banach space.

A final definition which will be of use to us is the following.

Definition 2.6 Let $\left(W,\|\cdot\|_{W}\right)$ and $\left(X,\|\cdot\|_{X}\right)$ be normed spaces, with $W$ a vector subspace of $X$. The closed unit ball $\mathbf{B}_{W}$ is said to be definably compactly contained in the closed unit ball $\mathbf{B}_{X}$ if every definable curve contained in $\mathbf{B}_{W}$ has a limit, with respect to the $\|\cdot\|_{X}$-norm, lying in $\mathbf{B}_{X}$.

\section{Convergence Results}

\subsection{Main Theorem}

The results in this section concern definable curves in various subspaces of $\mathcal{B}((0,1))$ and so throughout $\mathcal{F}$ is tacitly assumed to be a definable curve $\left\{F_{u}:(0,1) \rightarrow M \mid u \in(0,1)\right\}$ 
in $\mathcal{B}((0,1))$. However, this is merely a restriction for convenience. These results are all straightforwardly translated to the following two settings. First, they will apply with any bounded domain $Y$ in place of $(0,1)$, by o-minimality. Our results also apply to definable curves of maps $F_{u}:(0,1) \rightarrow M^{N}$, for $N>1$, after first making analogous definitions of the function spaces described in Examples 2.1; the only adjustment is to take throughout the modulus of a function $f=\left(f_{1}, \ldots, f_{N}\right)$ at $y \in(0,1)$ to be $\max _{1 \leq i \leq N}\left\{\left|f_{i}(y)\right|\right\}$. It is these maps which appear in the proof of the Pila-Wilkie Reparameterization Theorem (see [2], Section 4), for which the analogous version of Corollary 3.7 below is required.

The main result is the following.

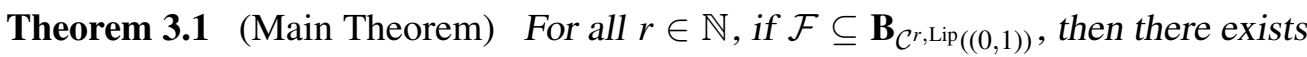
$F_{0} \in \mathbf{B}_{\mathcal{C}^{r, \operatorname{Lip}((0,1))}}$ such that $\left\|F_{u}-F_{0}\right\|_{r} \rightarrow 0$ as $u \rightarrow 0^{+}$; in particular, $\mathbf{B}_{\mathcal{C}^{r, \operatorname{Lip}((0,1))}}$ is definably compactly contained in $\mathbf{B}_{\mathcal{C}^{r}((0,1))}$.

In order to prove Main Theorem 3.1, we shall first need to prove the following straightforward lemmas.

Lemma 3.2 Let $\mathcal{F}$ be a subset of $\mathbf{B}_{\mathcal{B}((0,1))}$. Then there exists a definable function $F_{0}:(0,1) \rightarrow M$ in $\mathbf{B}_{\mathcal{B}((0,1))}$ such that $F_{u} \rightarrow F_{0}$ pointwise (i.e. $F_{u}(v) \rightarrow F_{0}(v)$ as $u \rightarrow 0^{+}$, for every $\left.v \in(0,1)\right)$.

Proof For each $v \in(0,1)$, the map $u \mapsto F_{u}(v)$ is definable, so $\lim _{u \rightarrow 0^{+}} F_{u}(v)$ exists, by o-minimality, and lies in $M \cup\{ \pm \infty\}$.

Moreover, for all $v \in(0,1)$,

$$
\left|\lim _{u \rightarrow 0^{+}} F_{u}(v)\right| \leq \sup _{u \in(0,1)}\left|F_{u}(v)\right| \leq \sup _{u \in(0,1)}|| F_{u} \|_{\infty} \leq 1 .
$$

Let $F_{0}:(0,1) \rightarrow M$ be given by $F_{0}(v):=\lim _{u \rightarrow 0^{+}} F_{u}(v)$. This is definable and we have that $\left|F_{0}(v)\right| \leq 1$, for all $v \in(0,1)$. Hence $\left\|F_{0}\right\|_{\infty} \leq 1$ and, by definition, $F_{u}$ tends to $F_{0}$ pointwise as $u \rightarrow 0^{+}$, as required.

Lemma 3.3 Suppose now that $\mathcal{F}$ is a subset of $\mathbf{B}_{\mathcal{C}^{0, \operatorname{Lip}((0,1))}}$. In that case, $F_{0}$, the pointwise limit defined as in Lemma 3.2, is also in $\mathbf{B}_{\mathcal{C}^{0, \operatorname{Lip}}((0,1))}$. Moreover, we have that $\left\|F_{u}-F_{0}\right\|_{\infty} \rightarrow 0$ as $u \rightarrow 0^{+}$.

Proof First note that $F_{0}$, being definable, is continuous at all but finitely many $v \in$ $(0,1)$, by the Monotonicity Theorem. 
Let $v_{1}, v_{2} \in(0,1)$ and let $\epsilon>0$. Then note that

$$
\left|F_{0}\left(v_{1}\right)-F_{0}\left(v_{2}\right)\right| \leq\left|F_{0}\left(v_{1}\right)-F_{u}\left(v_{1}\right)\right|+\left|F_{u}\left(v_{1}\right)-F_{u}\left(v_{2}\right)\right|+\left|F_{u}\left(v_{2}\right)-F_{0}\left(v_{2}\right)\right| .
$$

Choose $\delta>0$ sufficiently small that, whenever $u \in(0, \delta)$, the inequality

$$
\left|F_{u}\left(v_{i}\right)-F_{0}\left(v_{i}\right)\right|<\frac{\epsilon}{2}\left|v_{1}-v_{2}\right|
$$

holds for both $i=1$ and 2 .

Since $\left|F_{u}\left(v_{1}\right)-F_{u}\left(v_{2}\right)\right| \leq\left|v_{1}-v_{2}\right|$, as $F_{u} \in \mathbf{B}_{\mathcal{C}^{0, \operatorname{Lip}((0,1))}}$, we obtain

$$
\left|F_{0}\left(v_{1}\right)-F_{0}\left(v_{2}\right)\right| \leq(\epsilon+1)\left|v_{1}-v_{2}\right| \text {. }
$$

This is true for all $\epsilon>0$, and therefore

$$
\left|F_{0}\left(v_{1}\right)-F_{0}\left(v_{2}\right)\right| \leq\left|v_{1}-v_{2}\right| .
$$

Thus $F_{0}$ is Lipschitz continuous, with Lipschitz constant $\left\|F_{0}\right\|_{\text {Lip }} \leq 1$. By Lemma 3.2, $\left\|F_{0}\right\|_{\infty} \leq 1$, so $F_{0}$ lies in $\mathbf{B}_{\mathcal{C}^{0, \operatorname{Lip}((0,1))}}$, which proves the first statement.

Suppose now that $\left\|F_{u}-F_{0}\right\|_{\infty} \nrightarrow 0$ as $u \rightarrow 0^{+}$, which is to say that $\sup _{v \in(0,1)}\left|F_{u}(v)-F_{0}(v)\right| \nrightarrow 0$ as $u \rightarrow 0^{+}$.

In this case, there would exist an $\eta>0$ for which the set

$$
\left\{u \in(0,1) \mid \exists v \in(0,1) \text { such that }\left|F_{u}(v)-F_{0}(v)\right| \geq \eta\right\}
$$

contains arbitrarily small values in $(0,1)$. This set is definable and so, by o-minimality, would contain an interval $\left(0, u_{\eta}\right)$. Moreover, by the property of definable Skolem functions together with the Monotonicity Theorem, there would exist a definable, continuous map $v^{*}:\left(0, u^{*}\right) \longrightarrow(0,1)$ on some subinterval $\left(0, u^{*}\right) \subseteq\left(0, u_{\eta}\right)$ such that, for all $u \in\left(0, u^{*}\right)$,

$$
\left|F_{u}\left(v^{*}(u)\right)-F_{0}\left(v^{*}(u)\right)\right| \geq \eta .
$$

Suppose that such a function $v^{*}$ exists and fix $\epsilon \in(0, \eta)$. The continuity of $v^{*}$ implies that there exists some $u_{1} \in\left(0, u^{*}\right)$ such that, for all $u \in\left(0, u_{1}\right)$,

$$
\left|v^{*}(u)-v^{*}\left(u_{1}\right)\right|<\frac{\eta-\epsilon}{2} .
$$

Since $F_{u} \rightarrow F_{0}$ pointwise, there is some $u_{2} \in\left(0, u_{1}\right)$ such that, if $u \in\left(0, u_{2}\right)$, then

$$
\left|F_{u}\left(v^{*}\left(u_{1}\right)\right)-F_{0}\left(v^{*}\left(u_{1}\right)\right)\right|<\epsilon .
$$


Hence, if $u \in\left(0, u_{2}\right) \subseteq\left(0, u_{1}\right) \subseteq\left(0, u^{*}\right)$, then, combining (3.3.1), (3.3.3) and (3.3.2), we see that

$$
\begin{aligned}
\eta-\epsilon & <\left|F_{u}\left(v^{*}(u)\right)-F_{0}\left(v^{*}(u)\right)\right|-\left|F_{u}\left(v^{*}\left(u_{1}\right)\right)-F_{0}\left(v^{*}\left(u_{1}\right)\right)\right| \\
& \leq\left|F_{u}\left(v^{*}(u)\right)-F_{u}\left(v^{*}\left(u_{1}\right)\right)\right|+\left|F_{0}\left(v^{*}(u)\right)-F_{0}\left(v^{*}\left(u_{1}\right)\right)\right| \\
& \leq 2\left|v^{*}(u)-v^{*}\left(u_{1}\right)\right|, \text { as } \mathcal{F} \cup\left\{F_{0}\right\} \subseteq \mathbf{B}_{\mathcal{C}^{0, \operatorname{Lip}}((0,1))}, \\
& <\eta-\epsilon,
\end{aligned}
$$

which is clearly a contradiction.

Thus $\left\|F_{u}-F_{0}\right\|_{\infty} \rightarrow 0$ as $u \rightarrow 0^{+}$, as required.

We may now build on these lemmas with the following central proposition.

Proposition 3.4 Let $\mathcal{F}$ be a subset of $\mathbf{B}_{\mathcal{C}^{1, \operatorname{Lip}}((0,1))}$. There exists $H_{0} \in \mathbf{B}_{\mathcal{C}^{0, \operatorname{Lip}}((0,1))}$ such that $\left\|F_{u}^{\prime}-H_{0}\right\|_{\infty} \rightarrow 0$ as $u \rightarrow 0^{+}$. Moreover, $F_{0}$, the pointwise limit of $\mathcal{F}$, is differentiable on $(0,1)$ and $F_{0}^{\prime}=H_{0}$, so we may conclude that $F_{0}$ lies in $\mathbf{B}_{\mathcal{C}^{1, \operatorname{Lip}}((0,1))}$ and $\left\|F_{u}-F_{0}\right\|_{1} \rightarrow 0$ as $u \rightarrow 0^{+}$.

Proof Consider the parameterized family $\mathcal{F}^{\prime}:=\left\{F_{u}^{\prime} \mid F_{u} \in \mathcal{F}\right\}$. Since $\mathcal{F}^{\prime}$ is contained in $\mathbf{B}_{\mathcal{C}^{0, \operatorname{Lip}((0,1))}}$, we may apply Lemmas 3.2 and 3.3 to $\mathcal{F}^{\prime}$ to see that $H_{0}$, the pointwise limit of $\mathcal{F}^{\prime}$, lies in $\mathbf{B}_{\mathcal{C}^{0, \operatorname{Lip}((0,1))}}$ and, moreover, $\left\|F_{u}^{\prime}-H_{0}\right\|_{\infty} \rightarrow 0$ as $u \rightarrow 0^{+}$.

Since $\mathbf{B}_{\mathcal{C}^{1, \operatorname{Lip}((0,1))}}$ is contained in $\mathbf{B}_{\mathcal{C}^{0, \operatorname{Lip}((0,1))}}$, we have, again by Lemma 3.3, that $F_{0} \in \mathbf{B}_{\mathcal{C}^{0, \operatorname{Lip}((0,1))}}$ and $\left\|F_{u}-F_{0}\right\|_{\infty} \rightarrow 0$ as $u \rightarrow 0^{+}$. Hence it is enough to prove that $F_{0}$ is differentiable and that $F_{0}^{\prime}=H_{0}$.

The fact that $\left\|F_{u}^{\prime}-H_{0}\right\|_{\infty} \rightarrow 0$ as $u \rightarrow 0^{+}$tells us that, for all $\epsilon>0$, there is some $\delta \in(0,1)$ such that, for any $u_{1}, u_{2} \in(0, \delta)$ and any $v \in(0,1)$,

$$
\left|F_{u_{1}}^{\prime}(v)-F_{u_{2}}^{\prime}(v)\right|<\frac{\epsilon}{4} \text {. }
$$

Now we may use the Mean Value Theorem to obtain the following estimate, for any $u_{1}, u_{2} \in(0, \delta)$, any $v \in(0,1)$ and any $h \in(-v, 1-v)$ : 


$$
\begin{aligned}
& \left|\frac{F_{u_{1}}(v+h)-F_{u_{1}}(v)-h F_{u_{1}}^{\prime}(v)}{h}-\frac{F_{u_{2}}(v+h)-F_{u_{2}}(v)-h F_{u_{2}}^{\prime}(v)}{h}\right| \\
& \leq\left|\frac{\left(F_{u_{1}}-F_{u_{2}}\right)(v+h)-\left(F_{u_{1}}-F_{u_{2}}\right)(v)}{h}\right|+\left|\left(F_{u_{1}}^{\prime}-F_{u_{2}}^{\prime}\right)(v)\right| \\
& \leq \sup _{\theta \in(0,1)}\left|\left(F_{u_{1}}^{\prime}-F_{u_{2}}^{\prime}\right)(v+\theta h)\right|+\left|\left(F_{u_{1}}^{\prime}-F_{u_{2}}^{\prime}\right)(v)\right| \\
& \leq \frac{\epsilon}{4}+\frac{\epsilon}{4}=\frac{\epsilon}{2} .
\end{aligned}
$$

So, if we let $u_{1} \rightarrow 0^{+}$, then we have that, for fixed $u_{2}, h$ and $v$,

$$
\left|\frac{F_{0}(v+h)-F_{0}(v)-h H_{0}(v)}{h}-\frac{F_{u_{2}}(v+h)-F_{u_{2}}(v)-h F_{u_{2}}^{\prime}(v)}{h}\right| \leq \frac{\epsilon}{2} .
$$

By the differentiability of $F_{u_{2}}$, we also have some $\delta^{\prime}>0$ with $\delta^{\prime}<v, 1-v$ such that, if $0<|h|<\delta^{\prime}$, then

$$
\left|\frac{F_{u_{2}}(v+h)-F_{u_{2}}(v)-h F_{u_{2}}^{\prime}(v)}{h}\right|<\frac{\epsilon}{2} .
$$

Therefore let $h$ be such that $0<|h|<\delta^{\prime}$ (with $u_{2}$ and $v$ as above). Then, by (3.4.4) and (3.4.5),

$$
\begin{aligned}
& \quad\left|\frac{F_{0}(v+h)-F_{0}(v)-h H_{0}(v)}{h}\right| \\
& \leq\left|\frac{F_{0}(v+h)-F_{0}(v)-h H_{0}(v)}{h}-\frac{F_{u_{2}}(v+h)-F_{u_{2}}(v)-h F_{u_{2}}^{\prime}(v)}{h}\right| \\
& \quad+\left|\frac{F_{u_{2}}(v+h)-F_{u_{2}}(v)-h F_{u_{2}}^{\prime}(v)}{h}\right| \\
& \leq \frac{\epsilon}{2}+\frac{\epsilon}{2}=\epsilon .
\end{aligned}
$$

Consequently, we may conclude that

$$
\left|\frac{F_{0}(v+h)-F_{0}(v)-h H_{0}(v)}{h}\right| \rightarrow 0 \text { as } h \rightarrow 0 ;
$$

or, in other words, that $F_{0}$ is differentiable on $(0,1)$, with $F_{0}^{\prime}$ equal to $H_{0}$, as required.

We may now easily outline the proof of the Main Theorem 3.1. 
Proof of Main Theorem 3.1 The aim is to prove the following statement by induction on $r$ :

For all $r \in \mathbb{N}$, if $\mathcal{F}$ is contained in $\mathbf{B}_{\mathcal{C}^{r, \text { Lip }}((0,1))}$, then the pointwise limit $F_{0}$ also lives in $\mathbf{B}_{\mathcal{C}^{r, \operatorname{Lip}}((0,1))}$, with $F_{0}^{(t)}(v)=\lim _{u \rightarrow 0^{+}} F_{u}^{(t)}(v)$, for all $v \in(0,1)$ and for all $t \in\{0, \ldots, r\}$, and, moreover, $\left\|F_{u}-F_{0}\right\|_{r} \rightarrow 0$ as $u \rightarrow 0^{+}$.

We already have all of the steps in place. Lemma 3.3 is the base case $(r=0)$. The inductive step is then given by an analogous argument to the proof of Proposition 3.4.

\subsection{Further Results}

There are many similar results of this nature which we may now obtain using Main Theorem 3.1. An example of these is given here, followed by a discussion of some others.

Proposition 3.5 If $\mathcal{F} \subseteq \mathbf{B}_{\mathcal{C}^{r, \operatorname{Lip}((0,1))}}$ is Cauchy in the $\|\cdot\|_{r, \mathrm{Lip}}-$ norm, i.e. $\left\|F_{u_{1}}-F_{u_{2}}\right\|_{r, \text { Lip }} \rightarrow 0$ as $u_{1}, u_{2} \rightarrow 0^{+}$, then, for the pointwise limit $F_{0}$, $\left\|F_{u}-F_{0}\right\|_{r, \text { Lip }} \rightarrow 0$, as $u \rightarrow 0^{+}$.

Proof Let us first note that the following two requirements are necessary, as $u$ tends to $0^{+}$, for $\left\|F_{u}-F_{0}\right\|_{r, \text { Lip }}$ to tend to 0 :

(i) $\left\|F_{u}^{(t)}-F_{0}^{(t)}\right\|_{\infty} \rightarrow 0$, for every $t=0, \ldots, r$;

(ii) $\left\|F_{u}^{(r)}-F_{0}^{(r)}\right\|_{\text {Lip }} \rightarrow 0$.

Since Main Theorem 3.1 tells us that (i) holds regardless of whether or not $\mathcal{F}$ is Cauchy in the $\|\cdot\|_{r, \text { Lip }}-$ norm, it will be sufficient to show that $\left\|F_{u}^{(r)}-F_{0}^{(r)}\right\|_{\text {Lip }} \rightarrow 0$ as $u \rightarrow 0^{+}$.

By considering the functions $\left(F_{u}-F_{0}\right)^{(r)}$, for each $u \in(0,1)$, we may simplify the argument slightly by supposing that $\mathcal{F}$ is a definable curve which consists of Lipschitz continuous functions $F_{u}$, each of which has $\left\|F_{u}\right\|_{\text {Lip }} \leq 1$, is such that $F_{u} \rightarrow 0$ pointwise as $u \rightarrow 0^{+}$and is Cauchy with respect to the $\|\cdot\|_{0, \text { Lip }}-$ norm. We shall then show that $\left\|F_{u}\right\|_{\text {Lip }} \rightarrow 0$ as $u \rightarrow 0^{+}$. 
Suppose that $\left\|F_{u}\right\|_{\text {Lip }} \nrightarrow 0$ as $u \rightarrow 0^{+}$. This is the same as the statement that

$$
\operatorname{Lip}\left(F_{u}\right):=\sup _{\substack{x \neq y \\ x, y \in(0,1)}}\left\{\left|\frac{F_{u}(x)-F_{u}(y)}{x-y}\right|\right\} \nrightarrow 0 \text { as } u \rightarrow 0^{+} .
$$

So there must exist $\zeta>0$ such that, for every $u \in(0,1)$, there is a $\hat{u} \in(0, u)$ such that $\hat{x} \neq \hat{y} \in(0,1)$ exist with

$$
\left|\frac{F_{\hat{u}}(\hat{x})-F_{\hat{u}}(\hat{y})}{\hat{x}-\hat{y}}\right| \geq \zeta .
$$

Moreover, by the Monotonicity Theorem and the property of definable Skolem functions, there is some $u^{\prime} \in(0,1)$ and a definable, continuous function $\lambda:\left(0, u^{\prime}\right) \longrightarrow(0,1)^{2}$ such that, if $\lambda(u)$ is denoted by the ordered pair $\left(x_{u}, y_{u}\right)$, then, for all $u \in\left(0, u^{\prime}\right)$,

$$
\left|\frac{F_{u}\left(x_{u}\right)-F_{u}\left(y_{u}\right)}{x_{u}-y_{u}}\right| \geq \zeta \text {. }
$$

Let $\epsilon \in(0, \zeta)$. By our reduction, $\mathcal{F}$ is Cauchy in the $\|\cdot\|_{0, \text { Lip }}$-norm, so there is some $\tilde{u} \in\left(0, u^{\prime}\right)$ such that, for all $u_{1}, u_{2} \in(0, \tilde{u}),\left\|F_{u_{1}}-F_{u_{2}}\right\|_{\text {Lip }}<\epsilon$.

Now fix $u_{1} \in(0, \tilde{u})$. We know that, for $\lambda\left(u_{1}\right)=\left(x_{u_{1}}, y_{u_{1}}\right)$,

$$
\left|\frac{F_{u_{1}}\left(x_{u_{1}}\right)-F_{u_{1}}\left(y_{u_{1}}\right)}{x_{u_{1}}-y_{u_{1}}}\right| \geq \zeta
$$

Moreover, since $u_{1} \in(0, \tilde{u})$, we have that, for every $u_{2} \in\left(0, u_{1}\right)$,

$$
\left|\frac{\left(F_{u_{1}}\left(x_{u_{1}}\right)-F_{u_{2}}\left(x_{u_{1}}\right)\right)-\left(F_{u_{1}}\left(y_{u_{1}}\right)-F_{u_{2}}\left(y_{u_{1}}\right)\right)}{x_{u_{1}}-y_{u_{1}}}\right|<\epsilon
$$

since $\left\|F_{u_{1}}-F_{u_{2}}\right\|_{\text {Lip }}<\epsilon$ and $x_{u_{1}} \neq y_{u_{1}}$. Therefore, for every $u_{2} \in\left(0, u_{1}\right)$,

$$
\begin{aligned}
\left|\frac{F_{u_{2}}\left(x_{u_{1}}\right)-F_{u_{2}}\left(y_{u_{1}}\right)}{x_{u_{1}}-y_{u_{1}}}\right| & \geq\left|\frac{F_{u_{1}}\left(x_{u_{1}}\right)-F_{u_{1}}\left(y_{u_{1}}\right)}{x_{u_{1}}-y_{u_{1}}}\right| \\
& -\left|\frac{\left(F_{u_{1}}\left(x_{u_{1}}\right)-F_{u_{2}}\left(x_{u_{1}}\right)\right)-\left(F_{u_{1}}\left(y_{u_{1}}\right)-F_{u_{2}}\left(y_{u_{1}}\right)\right)}{x_{u_{1}}-y_{u_{1}}}\right| \\
& >\zeta-\epsilon,
\end{aligned}
$$

by (3.5.6) and (3.5.7). So we have, for all $u_{2} \in\left(0, u_{1}\right)$,

$$
\left|F_{u_{2}}\left(x_{u_{1}}\right)-F_{u_{2}}\left(y_{u_{1}}\right)\right|>(\zeta-\epsilon)\left|x_{u_{1}}-y_{u_{1}}\right| .
$$

Recall now that $F_{u} \rightarrow 0$ pointwise, so there is some $u^{\prime \prime} \in\left(0, u_{1}\right)$ such that if $u \in\left(0, u^{\prime \prime}\right)$, 
then both

$$
\begin{aligned}
\left|F_{u}\left(x_{u_{1}}\right)\right|< & \left(\frac{\zeta-\epsilon}{2}\right)\left|x_{u_{1}}-y_{u_{1}}\right| \\
& \text { and } \\
\left|F_{u}\left(y_{u_{1}}\right)\right|< & \left(\frac{\zeta-\epsilon}{2}\right)\left|x_{u_{1}}-y_{u_{1}}\right| .
\end{aligned}
$$

Then fix $u_{2} \in\left(0, u^{\prime \prime}\right) \subseteq\left(0, u_{1}\right) \subseteq(0, \tilde{u}) \subseteq\left(0, u^{\prime}\right) \subseteq(0,1)$. We may now conclude that

$$
\begin{aligned}
(\zeta-\epsilon)\left|x_{u_{1}}-y_{u_{1}}\right| & >\left|F_{u_{2}}\left(x_{u_{1}}\right)\right|+\left|F_{u_{2}}\left(y_{u_{1}}\right)\right|, \text { by (3.5.9) and (3.5.10) } \\
& \geq\left|F_{u_{2}}\left(x_{u_{1}}\right)-F_{u_{2}}\left(y_{u_{1}}\right)\right| \\
& >(\zeta-\epsilon)\left|x_{u_{1}}-y_{u_{1}}\right|, \text { by (3.5.8), }
\end{aligned}
$$

which is clearly a contradiction.

Thus we must have that $\left\|F_{u}\right\|_{\text {Lip }} \rightarrow 0$ as $u \rightarrow 0^{+}$, and hence that the definable curve $\mathcal{F}$ in the original statement of the theorem converges to $F_{0}$ in the $\|\cdot\|_{r, \mathrm{Lip}}-$ norm, as required.

Corollary 3.6 The space $\mathcal{C}^{r \text { Lip }}((0,1))$ is an $\mathcal{M}$-Banach space.

Proof If $\mathcal{F}$ is Cauchy in the space $\mathcal{C}^{r \text { Lip }}((0,1))$, then it is bounded in some closed ball of identifiable radius $q \geq 0$. Thus we may assume, without loss of generality, that

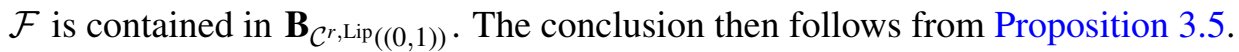

Finally, we come to the corollary indicated in Section 1, a form of which (for maps into cartesian products of $M$, as outlined at the start of this section) is used in the proof of the Reparameterization Theorem of [2].

Corollary 3.7 For all $r \geq 1$, the space $\mathbf{B}_{\mathcal{C}^{r}((0,1))}$ is definably compactly contained in $\mathbf{B}_{\mathcal{C}^{r-1}((0,1))}$.

Proof By the Mean Value Theorem, $\mathbf{B}_{\mathcal{C}^{r}((0,1))} \subseteq \mathbf{B}_{\mathcal{C}^{r-1, \operatorname{Lip}((0,1))}}$. Consequently, if $\mathcal{F}$ is a definable curve contained in $\mathbf{B}_{\mathcal{C}^{r}((0,1))}$, then, by Main Theorem 3.1, it has a limit $F_{0}$ in $\mathbf{B}_{C^{r-1, \operatorname{Lip}((0,1))}}$ with $\left\|F_{u}-F_{0}\right\|_{r-1} \rightarrow 0$ as $u \rightarrow 0^{+}$. 


\section{Final remarks}

In introducing a theory of definable Banach spaces, this paper addresses only the case of explicitly given spaces of one variable functions. This is sufficient to provide the details behind the key step in the proof of the Reparameterization Theorem of Pila and Wilkie [2] indicated in the Introduction (i.e. that reparameterizations converge to reparameterizations with sufficient differentiability). However, it should be relatively straightforward to extend these ideas to spaces of functions in a higher number of variables. Beyond that, the next natural step would be to consider how best to develop a theory of definable Banach spaces in greater generality. In particular, it would be of interest to develop the richest theory possible, and therefore to do so in a way which incorporates, for example, definable notions of the bounded linear operator and the dual space. Perhaps, rather than considering normed spaces, it would be most useful to consider spaces equipped with seminorms and to develop a theory in that context. It would certainly be interesting to see which results could be obtained definably over o-minimal expansions of real closed fields; there is the potential for a very rich theory to be uncovered here.

\section{Acknowledgements}

Thanks to A. J. Wilkie, under whose supervision this work was completed as part of the author's D.Phil. thesis [3] at the University of Oxford. Thanks also to Assaf Hasson for suggesting a simplification to the proof of Main Theorem 3.1 and to referees for their careful reading and various suggestions which have improved the presentation. This work was funded in part by the UK EPSRC, The Fields Institute for Research in Mathematical Sciences, University of Toronto, Canada, and the Zukunftskolleg, Universität Konstanz, Germany.

\section{References}

[1] L van den Dries, Tame Topology and O-Minimal Structures, London Mathematical Society Lecture Note Series 248, Cambridge University Press, 1998; doi: 10.1017/CBO9780511525919.

[2] J Pila, A J Wilkie, The rational points of a definable set, Duke Math. J. 133 (2006) 591-616; doi: 10.1215/S0012-7094-06-13336-7. 
[3] ME M Thomas, Convergence and Parameterization in O-Minimal Structures, D.Phil. Thesis, University of Oxford, 2009.

Zukunftskolleg, Fachbereich Mathematik und Statistik, Fach 216, Universität Konstanz, D78457 Konstanz, Germany

margaret . thomas@wolfson . oxon . org

Received: 3 November $2011 \quad$ Revised: 13 December 2011 